\title{
Zur Frage der Kontagiosität der Encephalitis lethargica epidemica ${ }^{1}$ ).
}

\author{
Von \\ Privatdozent Dr. Georg Stiefler. \\ (Eingegangen am 1. Oktober 1921.)
}

Die Encephalitis lethargica (E. 1.) ist, wie das ihr gegebene Beiwort „epidemica" besagt, durch ein ausgesprochen epidemisches Auftreten charakterisiert; sie hat als eine wahre Seuche fast den ganzen Erdball ergriffen und ist in verschiedenen Ländern und Gegenden in so ausgedehnten und örtlich dichten Epidemien aufgetreten, wie ähnliches hinsichtlich epidemischer Erkrankungen des Zentralnervensystems wohl nur bei der spinalen Kinderlähmung beobachtet worden ist. Wie bei letzterer kommt als Ursache der E. I. ein belebter Mikroorganismus in Frage, und es ergibt sich daraus für uns die Aufgabe, nach den Quellen und Wegen der Infektion zu suchen und vor allem nachzuforschen, ob die E. 1. eine kontagiöse Erkrankung ist, die von Mensch zu Mensch übertragen wird, wie dies bei der spinalen Kinderlähmung zuerst Wick man anläßlich der großen schwedischen Epidemie im Jahre 1905 durch seine vorbildlichen, in ihrer Vollkommenheit unerreichten Untersuchungen eindeutig festgestellt hat. Bei Durchsicht der vorliegenden Literatur der E. l. finden wir zunächst bei v. Econo mo, der das Krankheitsbild der E. 1. als selbständige klinische Einheit geschaffen hat, daß Fälle von Hausinfektion oder Erkrankung an Geschwistern, Ehepaaren und Stubengenossen nie beobachtet wurden, weshalb v. Economo den völligen Mangel einer Utbertragbarkeit der E. l. von Person zu Person betont. In der Mehrzahl der später erschienenen Arbeiten, deren Material den zahlreichen Epidemien des Jahres 1920 entstammt, wird die Frage der Kontagiosität überhaupt nicht berührt oder nur ab und zu in gleichfalls ablehnendem Sinne kurz beantwortet ${ }^{2}$ ). So sahen Umber, Piotrowski keine Verbreitung der E. l. in den Krankensälen weder bei Kranken noch beim Pflegepersonal, Co m b y keinen Fall von Kontagion.

1) Nach einem am 11. Oktober 1921 im Verein für Psychiatrie und Neurologie in Wien gehaltenen Vortrage.

2) Anmerkung bei der Korrektur: v. Economo wies allerdings erst kürzlich darauf hin, daß der Befund des Virus im Nasenrachenraum zur Vorsicht mahne und an die Möglichkeit der Ubertragung durch Taschentücher denken lasse (Wiener med. Wochenschr. 1921, Nr. 30). 
Grünewald hebt in seinem Sammelreferat hervor, daß die E. J. von Mensch zu Mensch nicht übertragbar sei, Encephalitisfälle zu anderen Patienten gelegt werden können, ohne daß für dieselben die Gefahr der Kontaktinfektion bestünde. E. Müller konnte in seinen Fällen eine Ubertragung der E. 1. von Person zu Person und durch anscheinend gesunde Zwischenträger nicht feststellen, er betont aber, „daß solche negativen Ergebnisse nur mit großer Vorsicht zu verwerten sind, die Schwierigkeiten des Zwischenträgernachweises außerordentlich groß seien, ein einziger positiver Fall unendlich mehr bedeute als zahlreiche negative".

Die einschlägige Literatur weist aber auch eine bereits ganz stattliche Anzahl vorwiegend aus Frankreich stammender gegenteiliger Beobachtungen auf, die dafür sprechen, daß die E. 1. zu den als kontagiös anerkannten Infektionskrankheiten gehört. H. W. Maier gibt in seiner Arbeit einen kleinen Auszug des im Bulletin mensuel des „Office international d'hygiène publique" erschienenen ausführlichen Berichtes über das epidemische Auftreten der E. l. in England und zum Teil auch in Frankreich, dem zu entnehmen ist, daß in England nach Einführung der Anzeigepflicht 338 Fälle gemeldet wurden, wovon 126 als sichere E. l. betrachtet werden konnten. Hierunter fand sich eine Beobachtung von familiärer Erkrankung, 3 Fälle betreffend, die in der gleichen Wohnung nacheinander am 19. April, 4. und 8. Mai erkrankten. In dem erwähnten Berichte wird die große Seltenheit der direkten Ubbertragung betont und hierbei auf den letal endigenden Fall eines Mädchens gewiesen, das mit 4 Geschwistern im gleichen Bette schlief, wobei dieselben gesund belieben. H. W. Maier sah bei der Züricher Epidemie unter seinen Fällen ein mal eine familiäre Erkrankung (2 Fälle in der gemeinsamen Wohnung) und führt weiterhin aus, ,daß über die Hälfte der von. ihm mitgeteilten Fälle Eisenbahnangestellte, deren Angehörige oder vor der Erkrankung mit dem Fremdenverkehr in direkter Berührung waren. Netter beschäftigte sich an der Hand eines großen Beobachtungsmaterials eingehend mit dem Studium der Kontagiosität der E. I. und fand, daß dieselbe in der Regel gering, nur in 4,6\% seiner Fälle vorhanden war; er bringt eine größere Anzahl recht überzeugender Einzelbeispiele für die unzweifelhaft vorhandene Kontagiosität der E. l. und betont, daß selbst klinisch längst abgelaufene Fälle, weiterhin die gesunde Umgebung des Kranken, vielleicht auch Gegenstände das Virus verbreiten können. Netter empfiehlt möglichst lange, bis in die Rekonvaleszenz hinein, dauernde Isolierung der Kranken, Desinfektion der Kleider, Wäsche, des Krankenzimmers sowie Festsetzung der Anzeigepflicht. Die Aufnahme des Virus erfolgt nach Netter durch die Nasenhöhle, die Ausscheidung durch Nasen- und Mundhöhle. Netter teilt an anderer Stelle noch 2 Fälle von typischer E. 1. mit, deren Brüder 
8 bzw. 14 Tage vorher an epidemischem Singultus erkrankt waren, und berichtet über 2 weitere analoge Fälle anderer Beobachter. Hier. wäre auch der Fall von Le moine zu erwähnen, der, fern von seinen Angehörigen wohnend, an mehrtägigem fieberhaften Singultus erkrankt war, einen Tag später zu seiner Familie zurückkehrte, wo nach 5 Tagen sein jüngerer Bruder an typischer E. l. erkrankte. Le mierre beschreibt einen Fall von familiärer Erkrankung an E. 1., der die Annahme einer direkten Übertragung sehr wahrscheinlich macht: Ein älterer Mann erkrankte im Dezember 1920 an E. 1., seine Tochter hatte Febr. 1918 eine Encephalitis durchgemacht, die aber nicht vollkommen abheilte und im Spätherbst 1920 neuerliches Aufflackern der Symptome darbot. Nach Lemierre sind als Virusträger insbesondere die prolongierten Formen anzusehen, die zur kalten Jahreszeit häufig akute Schübe aufweisen und dadurch eine erhöhte Kontagiosität zeigen, während anderseits die Gesunden zur gleichen Jahreszeit eine besondere Empfindlichkeit für das Virus zu haben scheinen. Claude und de Laulerie berichteten 2 im Krankenhaus entstandene Fälle von epidemischer Encephalitis (Auftreten derselben nach Aufnahme je eines frischen Falles von E. 1. in die beiden Krankensäle); in der sich anschließenden Diskussion teilte Netter 2 einschlägige Fälle von. Ansteckung durch alte, nicht völlig ausgeheilte Fälle mit, Ha lle den Fall einer an E. l. erkrankten, ihr Kind stillenden Mutter, das ungefähr 10 Tage später unter ähnlichen Erscheinungen erkrankte und bald darauf starb, wobei er die Frage offen ließ, ob die Virusübertragung durch die Milch oder den Speichel erfolgte, weiterhin brachte Lorta t-Ja kob Beispiele für die Verbreitung der E. 1. durch ganz leichte und rekonvaleszente Fälle. Roger und Blanchard sahen bei 2 Korporalschülern, die demselben Zuge angehörten, viel miteinander verkehrten und zusammen schliefen, im Abstande von 14 Tagen Erkrankung an E. 1. und berichteten weiterhin über die bisher in der Liberatur niedergelegten, bereits ziemlich reichhaltigen Erfahrungen von Kontagiosität der E. 1., wonach die Grenzen der Inkubationszeit ziemlich weite sind (14 Tage bis 3 Monate) und als Virusträger in erster Linie die Formes frustes, aber vermutlich auch die chronischen Verlaufsformen in Betracht kommen; sie erwähnen als eine gewiß sehr lehrreiche Beobachtung aus der Literatur die Erkrankung von 12 unter 21 Insassen einer englischen Pension, die innerhalb einer Wocke erkrankten. Pecori, der die Encephalitisepidemie in Rom 1919/20 (347 Fälle) zu beobachten Gelegenheit hatte, bringt Beispiele, welche eindeutig für die Kontagiosität der E. 1. sprechen, wenn dieselbe auch als gering bezeichnet werden muB; die Inkubationszeit würde nach seinen Erfahrungen zwischen 4 und 15 Tagen schwanken. Auch Bakke fand bei der norwegischen Epidemie 1919/20 kontagiöse Fälle. Die schwedischen Autoren Kling und Liljenquist haben die epidemische 
Ausbreitung der E. 1. in dem sehr dünn bevölkerten Kirchspiel Vilhelmina (Lappland) erforscht und sind hierbei zu dem Ergebnis gekommen, daß die Übertragung von Person zu Person geschieht, wobei die Inkubationsdauer in 3 Fällen mit 10 Tagen bemessen werden konnte. Als Virusüberträger kommen insbesondere die rein katarrhalischen Fälle mit Störung des Allgemeinbefindens ohne cerebrale Symptome in Frage. Tierexperimentell konnte das Virus im Nasenrachensekrete und Darminhalte nachgewiesen werden, diese kommen auch für die Ausscheidung des Virus in Betracht. Do pter hält gleichfalls die Verbreitung der E. l. durch unausgebildete Fälle für sehr wahrscheinlich und wies erst kürzlich wieder darauf hin, daß die Geographie der Seuchengänge der E. l. sowie zahlreiche Einzelbeispiele (Abortivformen, Rekonvaleszente, gesunde Zwischenpersonen) für die Übertragbarkeit der E. l sprechen. Außer der eingangs erwähnten Beobachtung $\mathrm{H}$. W. Maiers konnte ich in der deutschen Literatur trotz der so zahlreich erschienenen Arbeiten Beobachtungen über kontagiöse Beziehungen der E. I. nicht auffinden; es wäre nur noch die Mitteilung H. Schlesingers zu erwähnen, der eine UUbertragbarkeit der ,Nervengrippe“ zwar nicht beobachtete, jedoch 2 mal bei Familienepidemien und wiederholt bei Spitalinfektionen sah, daß von einer Infektionsquelle aus (Lungengrippe) gleichzeitig verschiedene Personen erkrankten, die einen an Pneumonie, die anderen an Encephalitis ${ }^{1}$ ).

In den Jahren 1920 und 1921 konnten wir in Linz und Umgebung sowie auf dem Lande (Oberösterreich) epidemisches Auftreten der E. 1. feststellen, das zeitlich vorwiegend an die kalte Jahreszeit gebunden war, im Laufe des Januars begann, Mitte Februar seinen Höhepunkt erreichte und Ende März allmählich erlosch; im April und Mai wurden nur mehr vereinzelte Nachzügler beobachtet. Daß einzelne, sogar gehäufte Fälle in den letzten Jahren bei uns vorgekommen, aber nicht richtig gedeutet worden sind, kann heute als sehr wahrscheinlich angenommen werden. Ich selbst sah in den Jahren 1918 und 1919 mehrere sporadische Fälle, die ihrem klinischen Gepräge und Verlaufe, dem negativen (makroskopischen) Obduktionsbefunde nach durchaus dem Bilde der E. 1. entsprachen, und konnte anläßlich einer Umfrage bei Kollegen, z. B. von Herrn Prim. Dr. S pechtenha user, in Erfahrung bringen, da $\beta$ er im Winter 1917 in einer Ortschaft in der Nähe von Wels mehrere Fälle mit Fieber, Augenmuskellähmungen und Schlafsucht beobachtet hat, die nach der mir gegebenen Schilderung mit großer Wahrscheinlichkeit als E. 1. anzusehen sind.

1) Anmerkung bei der Korrektur: P. H. Kramer denkt hinsichtlich der Ausbreitung der Encephalitis lethargica an eine Ubertragung derselben durch zurückbleibende Viruszwischenträger (Zentralbl. f. d. ges. Neur. u. Psych. XXVI. 5. H.). 
Die Gesamtzahl der von mir, zum Teil mit Herm Kollegen v. K urz, untersuchten Fälle beträgt bisher 128, wovon in 72 der Verlauf der Erkrankung vom Beginn an verfolgt werden konnte. Aus diesem Material stammen folgende Beobachtungen, die meines Erachtens zugunsten der Auffassung der E. 1. als einer kontagiösen Erkrankung in Betracht kommen.

Beobachtung I.

Fall 1: Bei der 33 jährigen Kaufmannsgattin L. B. waren die einleitenden Symptome (1l. II. 1920) allgemeines Übelbefinden, Mattigkeit, sehr schmerzhafte Muskelzuckungen in den Bauchdecken, am Rücken und im Zwerchfell (Singultus), weiterhin bestand eine tonische Anspannung des rechten Parametriums, das sich bei der Untersuchung bretthart anfühlte und einen Tumor vortäuschte, sowie eine Erschwerung der Harnentleerung, die wiederholtes Katheterisieren nötig machte. Von seiten der Hirnnerven fand sich beiderseitige Ptosis, Pupillendifferenz, Abducens- und Facialisparese links, verwaschene, leise Sprache; delirante Züge und schläfriges Wesen, zuweilen deutliche Schlafsucht, wechselten beständig miteinander ab. Von Mitte März an durch einige Wochen anhaltende Besserung, dann neuerliche Verschlimmerung mit rasch fortschreitender allgemeiner Prostration. Pat. war im 6. Monate gravid, künstliche Unterbrechung der Schwangerschaft ersehien indiziert, wenige Stunden nach vorgenommenem Eingriff Exitus. Obduktion wurde verweigert. Die Temperatur war fast durchwegs eine leicht febrile, katarrhalische Erscheinungen seitens der oberen Luftwege fehlten (gemeinsam beobachtet mit Herm Dr. Hella uer; veröffentlicht Wien. klin. Wochenschr. 1920, Nr. 14, Fall 8).

Fall 2: Die Mutter der Kranken (Fall 1), die 72jährige A. W., die in einem von der Wohnung ihrer Tochter entfernten Stadtgebiete wohnte, besuchte ihre Tochter am 24. II., verweilte mehrere Stunden im Krankenzimmer, küßte sie auch wiederholt. 11 Tage später erkrankte sie ohne jegliche Vorboten an Flimmern vor den Augen, rascher Ermüdbarkeit der Augen beim Lesen, Doppeltsehen. Okulistisch (Dr. A mon) wurde festgestellt: Rechte Pupille weiter als linke, reagiert langsam und wenig ausgiebig auf Licht und Konvergenz, rechter M. rectus internus paretisch. Nach 8 Tagen waren die okulären Erscheinungen und die subjektiven Beschwerden vollkommen verschwunden.

Fall 1 stellt sich dar als eine E. 1. mit bulbärmyelitischen Symptomen und myoklonusartigen motorischen Reizerscheinungen, die nach mehrmonatigem Bestande der akuten Krankheitsphase letal verlaufen ist. Fall 2 ist eine typische abortive Form mit rein okulären Symptomen (einseitige partielle Okulomotoriusparese), die nach kaum einwöchigem Verlaufe restlos abheilte. Die Entstehung des Falles 2 ist wohl nicht anders deutbar, als daß die Erkrankung von der Tochter auf die Mutter im Wege persönlichen, direkten Kontaktes übertragen wurde; die Beobachtung ist auch insofern wertvoll, als sie die Bestimmung der Inkubationszeit ermöglicht: Die Tochter erkrankte am 11. Februar, wurde am 24. Februar - das einzigemal - von ihrer Mutter besucht, die 11 Tage später an abortiver Encephalitis erkrankte.

Beobachtung II.

Auf der medizinischen Abteilung (Oberarzt Dr. H. Kugler) des Spitales der barmherzigen Schwestern in Linz lagen in einem Krankenzimmer 4 frische Fälle 
von Encephalitis lethargica, die in der Zeit vom 14. I. bis I6. II. 1920 erkrankt waren und wenige Tage nach Beginn der Erkrankung in das Spital aufgenommen worden waren, wo Herr Kollege v. K urz und ich Gelegenheit hatten, die Fälle zu beobachten. Es handelte sich um typische, ziemlich schwere Encephalitisfälle, die sämtliche, abgesehen von der bekannten Buntheit und Mannigfaltigkeit des Symptomenbildes -- Pupillenstörungen, Augenmuskelnerven- und Facialisparese, undeutliche, monotone Sprache, Abschwächung und Differenz der Sehnenreflexe an den Beinen, leichtere motorische Reizerscheinungen teils myoklonischen, teils choreatischen Charakter $;$ - eine ausgeprägte Schlafsucht aufwiesen, wodurch.der Gesamteindruck, den man manchmal beim Betreten des Zimmers bekam, ein ganz eigenartiger war: Es herrschte vollkommene Stille, man hörte nur das regelmäßige Atmen der ruhig Schlafenden. Katarrhalische Erscheinungen seitens des Nasenrachenraumes und der Luftröhren-Bronchien fehlten.

Fall 3: Am 1. III. 1920 erkrankte die 34jährige Ordensschwester B., welche die Encephalitisfalle seit ihrer Aufnahme ins Spital pflegte, an Kopfschmerzen, Schlaflosigkeit, Mattigkeit, Fieber; sie versah ihren Dienst zunächst noch weiter, alsbald traten leichte choreatische Zuckungen an beiden Armen auf, die aber nur einige Tage zu sehen waren. In der Woche vom 7. bis 13. III. fühlte sich Pat. wohler, war aber schlaftrunken; am 14. III. wurde sie bettlägerig, klagte über heftige neuralgieartige Schmerzen im Hinterhaupt und an der Stirne, war manchmal leicht benommen, redete vor sich hin. Keine Doppelbilder; Bulbi weichen, wenn man Pat. sich selbst überläßt, mit Vorliebe gegen oben ab. Pupillen reagieren auf Licht nur in minimalem Ausmaß. Am 18. und 20. III. je $10 \mathrm{~g}$ Elektrargol. 22. III.: Rechter Bulbus weicht beim Blick geradeaus nach außen ab. Ausgesprochene Schlafsucht. 26. III.: Pat. schlummert vor sich hin, ist leicht erweckbar, dann orientiert. Große Mattigkeit, noch leichte Kopfschmerzen. 30. III.: Augenbewegungen frei, Pupillenreaktion prompt, keine Kopfschmerzen, noch vermehrtes Schlafbedürfnis, abendliche Temperatur 37,8. Am 6. IV.: Seit 2 Tagen fieberfrei, kommt außer Bette, schläft im Stehen ein. 5. V.: Pat. war 3 Wochen in einem Erholungsheim, macht seit heute wieder Dienst. Ab und zu ziehende Schmerzen in dèr Kreuzbeingegend und in den Beinen; bei Tage noch leicht schläfrig, sieht aber frischer aus. 31. V.: Pat. versieht andauernd ihren Dienst als Pflegerin, hat noch manchmal mit dem Schlaf zu kämpfen. 4. VII.: Beschwerdefı $\mathbf{i}^{1}$ ).

Im vorliegenden Falle ist die Annahme einer direkten Utbertragung der Erkrankung von den Encephalitiskranken auf die Pflegeschwester, die Annahme einer Hausinfektion wohl kaum zu umgehen: In einem Krankenzimmer lagen 4 frische Fälle von Lethargica, die in der Zeit vom 20. 1. bis 18. 2. 20 ins Spital aufgenommen worden waren; am 1. März erkrankte die Pflegeschwester, die Tag für Tag bei den Kranken weilte, an einer ganz typischen Encephalitis (neuralgieartige Schmerzen, leichte delirante Züge, Schlafsucht, Blickparese, Internusschwäche, Pupillenträgheit, Fieber), die nach ungefähr 2 Monaten abheilte.

\section{Beobachtung III.}

Fall 4: Am 20. I. 1921 wurde ich vom Herrn Kollegen Prim. Doberer zu dem 44 jährigen Übungsschullehrer A. B. gerufen, der am 2. I. ohne Vorboten an Frösteln, Übelbefinden, leichtem Fieber, Hinterkopfschmerz, Doppeltsehen,

1) Für Überlassung der Krankengeschichte sage ich Herm Kollegen v. K urz auch an dieser Stelle meinen besten Dank. 
leichter Nackensteifigkeit erkrankt war und den Verdacht einer Meningitis erweckt hatte. Die ersten Tage war er Tag wie Nacht sehr unruhig, drängte aus dem Bette, sprach zusammenhanglos, verwirrt, meist von seinen beruflichen Pflichten, wollte in die Schule gehen usw. Bei der Untersuchung fanden sich subjektiv: Klagen über heftige Kopfschmerzen im Hinterhaupt, Schwindelgefühle beim Sichaufrichten; objektiv: Temperatur 38,8, Klopfempfindlichkeit der Scheitelbeine und des Hinterhauptes; beiderseits leichte Ptosis, linke Pupille etwas weiter als rechte, Reaktion auf Licht und Konvergenz beiderseits wenig ausgiebig und langsam, Parese des rechten Abducens und des Facialis in allen 3 Ästen, Sprache undeutlich artikuliert, leise, gegen Ende des Satzes kaum verständlich. Keine Nackensteifigkeit. Keine Parese der Gliedmaßen, Haut- und Sehnenreflexe gleichmäßig, gut auslösbar. Kein Babinski. Kein Kernig. Psychisch ist Pat. gut ansprechbar, aber leicht ermüdbar, schlummersüchtig, schläft während der Untersuchung häufig ein. Katarrhalische Erscheinungen der oberen Luftwege fehlen. Liquor cerebrospinalis: Mäßiger Druck bei der Entleerung, alle Eiweißreaktionen negativ, keine Pleocytose, WaR. negativ. Therapeutisch kam in Anwendung eine Serie von 6 intravenösen Elektrargolinjektionen und 10 subcutanen Injektionen von Staphylokokkenvaccin. Nach einem anfangs recht wechselvollen Verlaufe allmählich zunehmende Besserung. Pat. kam 10. III. außer Bett, bei der Untersuchung am 14. III. waren irgendwelche örtliche Krankheitserscheinungen nicht mehr nachzuweisen; er bot das Bild eines postinfektiösen, nervösen Schwächezustandes mit gesteigerter Reizbarkeit, Schlaflosigkeit bei erhöhtem Schlafbedürfnis, rascher Ermüdbarkeit, leicht-depressiver Gemütsverstimmung. Innerliche Medikation von As-Fe-Strychnin. Nach weiteren 5 Wochen war Pat. wieder vollkommen hergestellt und berufsfähig. Andauernde Heilung (Untersuchung am 30. VIII.).

Fall 5: Am 23. I. -. 3 Wochen nach Beginn der Erkrankung des Vaters (Fall 4) 一traten bei der 17 jährigen Tochter Marie, die Handelsangestellte in einem Geschäfte war, Frösteln, allgemeines Unbehagen, Mattigkeit auf, in den nächsten Tagen folgten heftige Kopf- und Leibschmerzen, Brechreiz, Schweißausbrüche, Temperatur 38,4. Okuläre Symptome fehlten, hingegen ließ sich eine leichte periphere Facialislähmung nachweisen, die in einer minder ausgiebigen Querfaltung der Stirne beim Blick nach oben, einem unvollkommenen Lidschluß, in Senkung und mangelhafter Beweglichkeit des linken Mundwinkels bei kaum angedeuteter Nasenlippenfalte links zum Ausdrucke kam. Ausgesprochene vasomotorische Übererregbarkeit. Schläfriges, apathisches Wesen ohne deutliche Schlafsucht. Keine psychischen Störungen. Das Fieber dauerte durch 6 Tage an, die Facialisschwäche war nach 14 Tagen nicht mehr erkennbar. Gesamtdauer der Erkrankung 3 Wochen.

Auch bei dieser Beobachtung ist die Annahme einer Utbertragung der Erkrankung vom Vater auf die Tochter sehr wahrscheinlich, zum mindesten naheliegend. Es handelt sich um eine 5 köpfige Familie (Eltern, 3 Kinder), die 3 Zimmer bewohnte, und zwar schliefen die Eltern in einem, die beiden Töchter im 2., der Sohn im 3. Zimmer. Das Schlaf. zimmer der Eltern lag in der Mitte und wurde auch während der Erkrankung des Vaters von sämtlichen Familienmitgliedern betreten. Der Vater erkrankte am 2. I. an einer unter meningealen Erscheinungen einsetzenden typischen E. 1.; bei der 17 jährigen Tochter M. traten die Symptome erst 3 Wochen später auf, wobei es nicht zu einer vollen Entwicklung des Krankheitsbildes kam,die nachweisbaren Erscheinungen 
aber eindeutig fürdas Vorhandensein einer abortiven Form der Lethargica sprachen. Die kontagiöse Verbindung der beiden Fälle im Sinne einer familiären Erkrankung durch persönliche Übertragung ist wohl sehr wahrscheinlich, wenn wir auch die Möglichkeit nicht ausschließën können, daß die Erkrankung der Tochter, die in einem Geschäft mit regem Kundenverkehr angestellt war, auf eine außerhalb des Hauses gelegene Infektionsquelle zurückzuführen ist. Bei der naheliegenden ersteren Annahme käme hinsichtlich der Berechnung der Inkubationszeit ein Zeitraum bis zu 3 Wochen in Betracht.

\section{Beobachtung IV.}

Fall 6: Der 47 jährige Hilfsarbeiter der Schiffswerft F. L. wurde am 3. III. 1921 dem von mir geleiteten Ambulatorium für Nervenkranke des Krankenkassenverbandes zugewiesen mit dem okulistischen Befunde (Dr. Ler perger): Abducenslähmung links, manchmal auch Schwäche des rechten Abducens. Fundus normal. Pat. gab an, daß er am 1. III. plötzlich an Frostgefühl, Kopfschmerzen, Mattigkeit, Doppeltsehen erkrankt sei, sich gegenwärtig aber abgesehen des gelegentlichen Doppeltsehens, wieder wohl fühle und in die Arbeit gehen wolle. Ich sprach in Hinblick auf die herrschende Encephalitisepidemie und analoge Erfahrungen aus dem Vorjahre den Fall als abortive Form einer E. 1 . an und berichtete in diesem Sinne dem Fachkollegen. Mitte Juni kam Pat. neuerlich zu mir, er fühlte sich subjektiv beschwerdefrei, es bestand aber noch eine leichte, linksseitige Abducensparese, die sich nach weiteren 4 Wochèn vollkommen zurückbildete. Nach Mitteilung des Kranken hatte sich sein Zustand Mitte März wesentlich verschlimmert, es traten Fieber, heftige Kopfschmerzen auf, er phantasierte durch einige Tage, glaubte sich immer in der Werkstätte zu befinden, auch litt er durch 14 Tage an Muskelzuckungen am Bauche und an den Armen. Er sei über 5 Wochen bettlägerig gewesen, fühlte sich sehr matt und schläfrig. Seit 8 Tagen sei er wieder in der Arbeit. Pat. gab weiter an, daß in der Nachbarwohnung ein ähnlicher Erkrankungsfall vorgekommen sei, der nach hierauf eingeholter Mitteilung des beide Fälle behandelnden Arztes (Dr. Klauber) und nach persönlich gepflogenen Erhebungen nur als eine E. l. gedeutet werden konnte.

Fall 7: Es handelte sich um die 19jährige Kontoristin F. St., deren Wohnraum sich an die Wohnung des Falles 6 unmittelbar anschloß, wohl einen eigenen Eingang, aber einen gemeinsamen Korridor hatte; zwischen beiden Parteien bestand ein reger Verkehr. Am 24. II. nun kam die F. St. in die Wohnung des Falles 6 und teilte diesem persönlich mit, daß sie seit gestern über heftige Kopfschmerzen, Schnupfen und Halsschmerzen zu klagen habe; sie wurde am selben Tage bettlägerig, es entwickelte sich ein delirantes Zustandsbild, dem später ein mehrwöchiger tiefer Lethargus folgte. Der Fall heilte nach ungefähr 8 Wochen ab, ich fand die Kranke Ende Juni sowie auch Anfang September dieses Jahres völlig beschwerdefrei.

Daß es sich in beiden Fällen um leichte, wenn auch mehr minder vollausgebildete E.-l.-Fälle gehandelt hat, bedarf wohl kaum einer näheren Erörterung, Art und Aufeinanderfolge der in Kürze geschilderten Symptome, die gleichzeitig herrschende Epidemie sprach zwingend für diese Annahme. Auch die Kontagiosität, die direkte-persönliche Beziehung beider Erkrankungsfälle dürfte kaum in Zweifel gezogen werden können, zumal die bei dem als Infektionsquelle in Betracht 
kommenden Falle 7 bestandenen katarrhalischen Erscheinungen seitens der Nasen- und Rachenschleimhaut eine Ubertragung auf dem Wege der sogenannten Tröpfcheninfektion ohne weiteres nahelegten. Fall 7 erkrankte am 24. Februar, besuchte den Fall 6 am nächsten Tage; später fand kein Verkehr zwischen den beiden Familien statt. 7 Tage später traten bei Fall 6 die ersten Erscheinungen auf, so daß wir in diesem Falle die im allgemeinen nicht so häufig zu findende Gelegenheit hätten, die Inkubationsdauer mit 8 Tagen bemessen zu können. Die ursprüngliche Beurteilung des Falles 6 als abortive Form war insoferne eine irrtümliche, als es sich damals um den Beginn bzw. den Vorläufer des später vollentwickelten Symptomenbildes gehandelt hatte. Die Beobachtung ist auch deshalb lehrreich, weil sie uns dafür zu sprechen scheint, daß wir bei näherer, persönlicher Nachforschung der Entstehung anscheinend isolierter Fälle von E. l. doch ab und zu kontagiöse Beziehungen zu andern Fällen aufdecken können, wie dies auch bei den beiden folgenden Kranken der Fall gewesen ist.

\section{Beobachtung V.}

Fall 8: Die 51 jährige Beamtensgattin M. W. erkrankte am 14. XI. 1919 unter allgemeinem Unbehagen, leichtem Frösteln, Schwitzen plötzlich über Nacht an einer schweren rechtsseitigen peripheren Facialislähmung mit heftigen neuralgiformen Schmerzen in der rechten Gesichtshälfte, insbesondere in der Gegend hinter dem Ohre, die nach einigen Tagen verschwanden. Ob Fieber anfangs vorhanden war, ließ sich infolge Unterlassens der Temperaturmessung nicht feststellen; später (vom 3. Krankheitsstage an) bestand keines mehr. Der elektrische Befund (komplette EaR., mittelschwere Form) wies auf eine schwere Lähmung hin; erst nach Verlauf von 7 Monaten kehrte die erloschene indirekte Erregbarkeit wieder zurück, die galvanomuskuläre Zuckung blieb noch 2 weitere Monate träge. Die Lähmung bildete sich sehr langsam zurück und ist auch gegenwärtig noch in Spuren erkennbar (seichtere Querfaltung der Stirne rechts als links, fast vollkommener, aber kraftloser Lidschluß, rechte Gesichtshälfte bleibt bei lebhafterer Mimik deutlich zurück). Die Frau gab als Ursache der Lähmung Erkältung bzw. Einwirkung kühler Zugluft an; sie habe am Tage vor Eintritt der Lähmung eine Eisenbahnfahrt gemacht, wobei infolge Zerbrochenseins des Fensters die demselben zugewendete rechte Gesichtshälfte andauernd der Zugluft ausgesetzt war.

Fall 9: Der 69jährige Beamte J. A. erkrankte am 27. XI. 1919 plötzlich an einer Lähmung der linken Gesichtshälfte mit Flimmern vor den Augen und ziemlich heftigen Schmerzen in der hinteren Ohrgegend. Keine Störung des Allgemeinbefindens. Keine nachweisbare äußere Veranlassung. So ausgesprochen die Facialislähmung in den ersten Tagen war, so ergab doch die in der 2. Woche vorgenommene elektrische Untersuchung ein qualitativ normales Verhalten, das die Stellung einer günstigen Prognose ermöglichte. Nach einer Gesamtdauer von 4 Wochen war die Facialislähmung vollkommen abgeheilt. Am 20. I. 1921 erkrankte Pat. plötzlich mit dem Gefühle allgemeiner Mattigkeit, Übelkeit, Schwindel an Doppeltsehen; okulistisch (Dr. Ler perger) und neurologisch wurde eine linksseitige Abducenslähmung festgestellt, die nach 2 Wochen restlos abheilte.

Auf Grund der im Falle 8 gegebenen Anamnese sprach ich denselben als eine Erkältungslähmung an und war auch geneigt, die gleiche Ätio- 
logie dem Falle 9 zugrunde zu legen, wenn sich auch anamnestisch keine analogen Verhältnisse fanden. Innerlich befriedigt war ich mit dieser Annahme nicht, zumal damals in Linz ein gehäuftes Auftreten von peripheren Facialislähmungen beobachtet wurde, das den Gedanken an die Entstehung durch ein infektiöses Agens nahelegte. Die Untersuchung der Fälle gab keinerlei Hinweise auf eine organische Erkrankung des zentralen Nervensystems (Lues, Tuberkulose, multiple Sklerose, Meningitis) oder eine andere Infektionskrankkeit, in deren Verlaufe organisch-nervöse Alterationen vorzukommen pflegen. Sie als abortive Form einer spinalen Kinderlähmung anzusprechen, wie wir derartige Fälle bei früheren Epidemien dieser Erkrankung (1908/09, 1912) allerdings selten zu beobachten Gelegenheit hatten, ging nicht an, da zur gleichen Zeit von einem nur leichten Gehäuftsein von Poliomyelitisfällen nichts bekannt war. Erst die wenige Wochen später einsetzende Encephalitisepidemie brachte uns hinsichtlich der kurz vorher beobachteten Häufung der Facialislähmungen die aufschließende Klärung; wir haben ebenso wie andere Autoren im Verlaufe der beiden Encephalitisepidemien eine Reihe von klinisch recht bunten Formes frustes kennengelernt, darunter als relativ häufigen Befund isolierte periphere Facialislähmungen, und wir dürften daher kaum in der Annahme fehlgehen, daß auch die erwähnten Fälle nucleoperipherer Facialislähmung als Vorläufer der alsbald nachfolgenden Encephalitisepidemie anzusprechen sind. Daß zum mindesten der Fall 9 eine Polio-Encephalitis pontis darstellt, geht unzweifelhaft aus seinem weiteren Verlaufe hervor. Im November 1919 trat bei ihm die periphere Facialislähmung auf, Ende Januar 1921 -- zur Zeit der Wiederkehr und des Aufblühens der Encephalitisepidemie - setzte bei ihm als klinischer Ausdruck des geschädigten anatomisch so eng benachbarten Kerngebietes eine gleichzeitige Abducenslähmung ein, ein geradezu klassisches Beispiel des Rezidivierens der Encephalitis zur Zeit neuerlichen epidemischen Auftretens der Erkrankung, wie wir dies bei den chronisch verlaufenden Fällen einige Male beobachten konnten und dies auch von anderen Autoren wiederholt beschrieben worden ist; so spricht Roger von einem saisonartigen Aufflackern der prolongierten Encephalitisformen (reviviscence saisonière). Fall 9 ist allerdings dadurch besonders bemerkenswert, daß zwischen der erstmaligen Erkrankung (Facialislähmung) und dem Auftreten des Rezidivs (Abducenslähmung) ein mehr als einjähriger Zeitraum völliger Gesundheit lag. Was mich aber eigentlich veranlaßte, den Fall 8, so sehr auch seine Anamnese für eine refrigeratorische Schädigung spricht, die ja möglicherweise als Hilfsursache weiterhin angenommen werden könnte, als abortive E. 1. aufzufassen und beide Fälle den anderen Beobachtungen anzureihen, ist der Umstand, daß, wie ich später in Erfahrung bringen konnte, beide Fälle in einem Hause 
wohnten, sich fast täglich sahen, wenn sie auch miteinander keinen näheren gesellschaftlichen Verkehr hatten, so daß die Annahme einer kontagiösen Vermittlung doch recht nahe liegt. Unsere Fälle sind ein weiterer Beleg für die erst kürzlich von E. Müller auf Grund reichlicher eigener Erfahrungen aufgestellte Forderung, daß die Lehre der rheumatischen Facialislähmung einer Revision bedarf.

\section{Beobachtung VI.}

Fall 10: Der 46jährige Tischlermeister H. W. erkrankte am 25. I. 1920 plötzlich mit leichtem Fieber und Frösteln, allgemeiner Mattigkeit, an Doppeltsehen, das ärztlich (Dr. Himmel) auf eine Abducenslähmung zurückgeführt wurde die sich auf eine energische Salicylkur zunächst etwas besserte, nach 4 Tagen aber unter Zunahme des Fiebers, heftigen Kopfschmerzen, Anzeichen von Nackensteifigkeit, Schwindel wieder stärker hervortrat. Gleichzeitig wurde totale Pupillenstarre, Fehlen der K.S.R. und A.S.R., Ausscheidung von Zucker im Harn festgestellt. Psychisch bestand ein mildes Beschäftigungsdelirium mit maniakalischer Färbung, Gesichts-, Gehörs- und Tasthalluzinationen, das mit Schlummersucht abwechselte. Letztere trat nach Abklingen der einwöchigen deliranten Phase stärker hervor. Neben der Abducensparese entwickelte sich im Verlaufe der Erkrankung eine gleichseitige periphere Facialislähmung. Sämtliche örtliche Erscheinungen von seiten des Gehirns und Rückenmarks bildeten sich relativ rasch zurück, am 25. II. war der Kranke auch subjektiv beschwerdefrei und konnte seiner Arbeit wieder nachgehen. Nase, Rachen und Bronchien waren während des ganzen Verlaufes der Erkrankung freigeblieben von katarrhalischen Erscheinungen. Durch wiederholte spätere Untersuchung konnten wir uns von der dauernden Heilung des Falles überzeugen (gemeinsame Beobachtung mit Herrn Dr. He is er, veröffentlicht Wien. klin. Wochenschr. 1920, Nr. 14, Fall 1).

Fall 11: Die 12 jährige Waise F. W. erkrankte Mitte Februar 1920 mit Fieber, Kopfschmerzen, Brechreiz, Schlaflosigkeit, deliranter Verworrenheit; an das delirante Stadium schloß sich ein mehrwöchiger tiefer Lethargus. Parallelgehend mit dem allmählichen Nachlassen desselben entwickelten sich motorische Reizerscheinungen von choreatischem Gepräge, die vorübergehend ungemein lebhaft waren. Mitte Mai konnte die Kranke als rekonvaleszent betrachtet werden. Der Fall wurde dadurch bemerkenswert, daß sich bei ihm nach Abschluß der akuten Krankheitsphase allmählich eine hypophysäre Fettsucht entwickelte (gemeinsam beobachtet mit Herrn Direktor Dr. Angel). Fall 10 und 11 wohnten in zwei einander benachbarten Straßen, ihre Wohnhäuser grenzten aber nicht aneinander, waren ungefähr 400 Schritte voneinander entfernt. Fall 11 und die 12 jährige Tochter des Falles 10 besuchten nicht nur dieselbe Schule, sondern saßen auch in der gleichen Schulbank nebeneinander. Ein weiterer Verkehr bestand zwischen den Familien nicht. Ich habe Fall 11 im April 1920 das erstemal gesehen, weniger Tage später ihre Schulkollegin, die Tochter des Falles 10 untersucht; letztere bot keinerlei Anzeichen einer Erkrankung und soll auch nach Aussage ihrer Angehörigen und des Arztes zur Zeit der Erkrankung des Vaters ebenso wie ihre Geschwister und Mutter vollkommen gesund gewesen sein.

Fall 10 trug das klinische Bild einer E. l. an sich, das durch das Hervortreten eines tabiformen Symptomenkomplexes bemerkenswert war, wie wir dies in mehr minder vollkommener Ausbildung nicht so selten bei den Fällen unserer Epidemie sahen und worauf ich bereits an anderer Stelle hingewiesen habe. Fall 11 hinwiederum zeigte als 
hervorstechendes Symptom während der akuten Phase eine der Chorea minor ähnliche Bewegungsunruhe. In epidemiologischer Hinsicht erscheint uns die Annahme einer kontagiösen Verbindung beider Fälle im Sinne einer Ưbertragung durch gesunde Viruszwischenträger gerechtfertigt: Fall 10 erkrankte am 25. Januar und wurde seit Beginn der Erkrankung von seiner Frau gepflegt und täglich von allen seinen Kindern besucht. Seine 12jährige Tochter saß Tag für Tag mit der 12 jährigen F. W. (Fall 11) in der Schule nebeneinander; bei letzterer stellten sich die ersten Krankheitserscheinungen Mitte Februar ein.

\section{Beobachtung VII.}

Fall 12: Der 23jährige Bauerssohn J. P. erkrankte Mitte März 1920 mit leichtem Fieber, Kopfschmerzen, Doppeltsehen, Schlafsucht, die sich immer mehr vertiefte und mehrere Wochen anhielt. Später merkte man eine Unsicherheit der Hände beim Ergreifen eines Gegenstandes und der Beine beim Stehen und Gehen. Sein Zustand besserte sich allmählich, er konnte sogar bei der häuslichen Arbeit wieder etwas mit helfen. Im Januar 1921 akute Verschlimmerung des Zustandes. Ich sah den Kranken das erstemal am 19. VII. 1921 : Parese des rechten M. rectus internus, rechte Pupille weiter als linke, Reaktion auf Licht und Konvergenz beiderseits nur spurweise zu erzielen. Übrige Hirnnerven frei. Keine Lähmung der Extremitäten, leichte Unsicherheit beim Ergreifen eines Gegenstandes, rechts stärker als links, beim Finger-Nasen- wie Knie-Hackenversuch wiederholt geringes Abweichen vom Zielpunkte. Beim Stehen grobes Schwanken nach rückwärts, Gang ausgesprochen taumelnd. Sensibilität frei.

Fall 13: Die 11 jährige Bauerstochter A. H., die Juni 1919 eine mehrtägige fieberhafte Grippe durchgemacht hatte, sich aber später wieder vollkommen gesund fühlte, erkrankte am 10. II. 1921 an einem ganz ähnlich entwickelten und verlaufenden Symptomenbilde; ihre Familie bewohnte ein einsam gelegenes Bauerngehöft, das von dem des Falles 12 ungefähr eine halbe Gehstunde entfernt war. Der Verkehr zwischen den beiden Familien war kein besonders reger, es ist aber erwiesen, daß die 13jährige Schwester der A. H. (Fall 13) den erkrankten J. P. (Fall 12) Ende Januar 1921 besuchte und 10-12 Tage später bei ihrer Schwester die Erkrankung zum Ausbruche kam. Bei der Untersuchung am 16. VII. 1921 bot sie das ausgeprägte Bild einer cerebellaren Ataxie mit unsicheren Bewegungen der o. E., groben Schwanken und Taumeln beim Gehen und Stehen. Hirnnerven frei, keine Lähmungen, Hautreflexe gleichmäßig auslösbar, Sehnenreflexe an den Beinen lebhaft, kein Babinski. Sensibilität frei, Lagegefühl an den Zehen schwer gestört.

Beide Kranke boten das Symptomenbild einer akut entstandenen cerebellaren Ataxie mit chronischem Verlaufe, das im ersten Falle mit bulbärmyelitischen Erscheinungen kompliziert war. Ihre genetische Zugehörigkeit zur E. 1. dürfte trotz der bei dieser Erkrankung im allgemeinen mehr seltenen cerebellaren Lokalisation kaum in Zweifel gezogen werden können: Das Auftreten der Fälle zur Zeit der damals herrschenden Encephalitisepidemie, die Verschlimmerung des ersten Falles analog der früher erwähnten Beobachtung (Fall 9) zur kalten Jahreszeit (zur Zeit der Wiederkehr der Epidemie), die insbesondere im ersten Falle ausgesprochene Schlafsucht und bulbärmyelitischen 
Erscheinungen sichern wohl zur Genüge die gestellte Diagnose. Die Kontagiosität beider Fälle kann hinsichtlich des geschilderten Verkehres wohl als wahrscheinlich angenommen werden, wobei als Infektionsmodus die Utbertragung durch einen gesunden Viruszwischenträger - die ältere Schwester des Falles 13, die den erkrankten Fall 12 besuchte - in Betracht kommt und die Inkubationszeit mit 10 bis 12 Tagen bemessen werden könnte.

\section{Beobachtung VIII.}

Fall 14: Der 9jährige K. D. erkrankte anfangs März 1920 mit Schüttelfrost und Fieber, heftigen Kopfschmerzen, Doppeltsehen, war anfangs insbesondere nachts sehr unruhig, phantasierte und sprach ,verworrenes Zeug“" zusammen, verfiel dann in Schlafsucht; er war durch mehrere Monate bettlägerig und wurde später seiner Umgebung auffällig durch die gezwungene steife Haltung, die Langsamkeit der Bewegungen, die leise, oft kaum verständliche Sprache. Bei der Untersuchung am 16. IV. 1921 fand sich. beiderseits Ptosis (rechts stärker als links), Lichtträgheit der Pupillen, Parese des rechten Mundfacialis, maskenartiger Gesichtsausdruck, Speichelfluß; monotone, kraftlose Sprache, nach vorne geneigte, starre Rumpfhaltung, Flexionsstellung der Arme und Beine, Bewegungsarmut, Langsamkeit und Erschwerung der aktiven Bewegungen, deutlich fühlbarer Rigor bei passiven Bewegungen -... alles in allem das $\mathrm{Pa}^{n} \mathrm{kins}$ onsche Syndrom mit bulbärmyelitischen Erscheinungen, das vervollständigt durch den eigenartigen psychischen Torpor und die wiederholt beobachtete Neigung des Kranken zum Einschlafen das so häufig beobachtete klinische Zustandsbild der E. 1. in ihrem terminalen Stadium bildet.

Die Mutter des Pat. teilte mir auf Befragen mit, daß in einem 2 Stunden von ihrem Anwesen entfernten Bauerngehöft ein 11 jähriges Mädchen unter gleichen Erscheinungen erkrankt sei und auch gegenwärtig das gleiche Bild wie ihr Sohn biete. Die ärztliche Untersuchung des Mädchens (Fall 15) ergab tatsächlich, daß es sich bei ihr wie im Falle 14 um einen typischen Parkinsonismus als Restzustand einer E. l. handelt. Die weitere Nachforschung ergab, daß die Eltern des Falles 14 zu Ostern 1920 (4. IV.) die Familie des 11 jährigen Mädchens besuchten, das 8 Tage später an Encephalitis erkrankte. ।

Es handelt sich bei den letzten 2 Fällen um ausgesprochen chronische Formen von Encephalitis mit dem klinischen Gepräge des Parkinsonismus, wie wir ihn so häufig als Schlußbild der E. l. finden. Beide Kranke bewohnten 2 Wegstunden voneinander entfernte, isoliert gelegene Häuser; der 2. Fall erkrankte 8 Tage nach dem Besuche der Eltern des bereits erkrankten 1. Falles, so daß auch in dieser Beobachtung wie in den vorhergegangenen ein indirekter Kontakt der beiden Kranken im Wege der Ưbertragung durch die Eltern des Ersterkrankten als gesunde Viruszwischenträger wahrscheinlich wird. Bemerkenswert ist, daß bei den Beobachtungen VII und VIII beide Kontaktfälle das gleiche klinische Bild - cerebellarer Symptomenkomplex (Fälle 12 und.13), Parkinsonismus (Fälle 14 und 15) - aufweisen ${ }^{1}$ ).

1) Anmerkung bei der Korrektur: Mitte Oktober sah ich einen Fall von Parkinson bei einer 32jährig:n Lehrerin, die in März 1921 an einer typischen Lethargica erkrankt war; sie unterrichtete privat das 10 jährige 
Vorliegende klinisch-epidemiologische Erfahrungen sprechen wohl mit Entschiedenheit zugunsten der Auffassung der Úbertragung der E. l. von Mensch zu Mensch; in 4 Beobachtungen war die Ansteckung als eine unmittelbare anzunehmen, wobei es sich in 2 um eine familiäre Kontaktinfektion (Tochter-Mutter, Vater-Tochter) handelte, in den 2 anderen um eine Hausinfektion (Erkrankung der Pflegeschwester bzw. des Wohnungsnachbars). Die Beobachtung V zeigt die Erkrankung zweier abortiver Fälle in eine $m$ Hause; ob sie im Sinne eines direkten persönlichen Kontaktes zusammengehören, ist nicht nachzuweisen, aber wahrscheinlich, wenn auch die Möglichkeit einer anderen Entstehungsweise (unbekannte gemeinsame oder verschiedene Infektionsquelle) nicht abgelehnt werden kann. Die Inkubationszeit konnte in 2 für ihre Berechnung geeigneten Fällen mit 8 bzw. 11 Tagen bemessen werden. In 3 Beobachtungen war die Annahme eines indirekten Kontaktes durch dritte Personen als klinisch gesunde Viruszwischenträger sehr wahrscheinlich, wobei in einem Falle der gemeinsame Schulbesuch, in den beiden anderen der Besuch von Gehöft zu Gehöft die Ansteckung vermittelte; in 2 Fällen konnte die Inkubationszeit mit 8 bzw. 12 Tagen berechnet werden.

Von den 8 Fällen, die als Infektionsquelle in Betracht kamen, befanden sich zu dieser Zeit 6 in der akuten Krankheitsphase, 2 boten die akuten Rezidive chronischer Fälle mit mehr als einjähriger Verlaufsdauer dar, wovon einer klinisch bereits abgeheilt war. Die durch unsere Beobachtungen festgestellte Tatsache der Kontagiosität der E. l. läßt in Bestätigung der schon früher berichteten gleichen Erfahrungen anderer Autoren eine Reihe von hygienisch-sanitären Maßnahmen als berechtigt und nötig erscheinen, so möglichst lange Isolierung der Kranken, Desinfektion des Krankenzimmers, der Kleider und Wäsche, bleibende Festsetzung der Anzeigepflicht.

Die E. l. weist in klinischer, pathohistologischer wie epidemiologischer Hinsicht eine recht nahe Verwandtschaft mit der epidemischen Kinderlähmung auf, die durch den Nachweis ihrer Kontagiosität weiterhin gefestigt wird. Beide Erkrankungen sind sich epidemiologisch zunächst dadurch ähnlich, daß ihr epidemisches Auftreten abhängig ist von einer bestimmten Jahreszeit, im Gegensatze zur Grippe, die derartige gesetzmäßige Beziehungen nicht aufweist. Während die epidemische Kinderlähmung in ausgesprochener Weise eine Erkrankung des Sommers und Herbstes mit Bevorzugung der Monate August-September ist (Wickman, Römer, eigene Beobachtungen), tritt die E.1. fast ausschließlich in der kalten Jahreszeit auf, in den Monaten

Mädchen eines Beamten, bei dem Ende Januar die ersten Anzeichen einer E. 1. aufgetreten waren und der heute gleichfalls einen ausgeprägten striären Symptomenkomplex darbietet. 
Dezember bis April mit dem Höhepunkte der Morbiditätskurve im Februar-März.

Aus den Ergebnissen der einschlägigen experimentellen Literatur (v. Wiesner, Löwe u. Strauss, Hirschfeld, Levaditi u. Harvier, Noguchi u. Flexner, Ottolenghi, Micheli, Thalhimer, Dörr u. Schnabel, Kling u. Liljenquist), auf die hier nicht näher eingegangen werden kann, wissen wir, daß als Virus der E. l. ein filtrierbares Virus in Betracht kommt, das dem der Poliomyelitis sehr ähnlich ist, aber im Gegensatz zu diesem auch auf Kaninchen und Katzen übertragen wird. Die Aufnahme erfolgt durch die Nasenhöhle, die Ausscheidung durch die Nasen- und Mundhöhle im wesentlichen wie bei der Poliomyelitis! Hinsichtlich der Art der Entstehung der Infektion könnte man bei der E. l. in Analogie zur Poliomyelitis (Rö mer) eine Tröpfcheninfektion beim Sprechen, Husten, Niesen als möglich bzw. wahrscheinlich annehmen, zumal Inokulationsversuche mit Waschflüssigkeitsfiltraten aus der Nasenhöhle positiv ausgefallen sind.

Was nun die Frage der Kontagiosität der E. l. betrifft, so ist zunächst hervorzuheben, daß die von mehreren Autoren (siehe oben) auf Grund ihrer Beobachtungen betonten negativen Ergebnisse durchaus nicht gegen die Kontagiosität der Erkrankung sprechen müssen; erinnern wir uns doch der bei den zahlreichen ausgedehnten PoliomyelitisEpidemien gemachten klinisch-epidemiologischen Erfahrungen! Die spinale Kinderlähmung war bereits seit Jahrzehnten bei uns als endemische Erkrankung bekannt, den Ärzten diagnostisch zweifellos geläufiger als die E. l., und es bedurfte trotz ihres wiederholt beobachteten epidemischen Auftretens geraumer Zeit, bis die Anschauung über ihre Kontagiosität sich endlich allgemeine Geltung erworben hat. Nach Römer deuteten wohl einzelne ältere Beobachtungen (Cordier), das Vorkommen von Geschwister- und Familienerkrankungen, die Ausbreitung längs der Verkehrswege (Leegard) bis zu einem gewissen Grade auf die Kontagiosität der Poliomyelitis hin, die Mehrzahl der führenden Autoren (Medin, Zappert u. a.) neigte aber dem gegenteiligen Standpunkte zu; erst Wick man ist es 1905 gelungen, anläßlich der großen schwedischen Epidemie die Kontagiosität der Poliomyelitis als feststehende Tatsache nachzuweisen, wobei zu betonen ist, daß seine Erfolge in erster Linie zurückzuführen sind auf die für die Nachforschung äußerst günstigen örtlichen Verhältnisse - dünnbevölkerte Gegenden, einsam gelegene Pfarrschaften, zerstreut liegende Gehöfte und die unermüdliche Tätigkeit und Sorgfalt, womit er den einzelnen Fällen nachging und ihre kontagiösen Beziehungen, die vor allem in Betracht kommende Verbreitung der Erkrankung durch anscheinend gesunde Viruszwischenträger aufdeckte. Daß Großstädte, dichtbevölkerte, industrie- und verkehrsreiche Gegenden einer derartigen Nach- 
forschung, insbesondere der Feststellung des indirekten Kontaktes äußerst abträglich sind, liegt auf der Hand und erklärt uns z. B. die negativen Ergebnisse gelegentlich der Poliomyelitisepidemien in New. York, Wien (Zappert). Ich verweise auf meine eigenen Untersuchungen und die von Linchner-Mally, Löcker, anläßlich des epidemischen Auftretens der spinalen Kinderlähmung in Linz und Oberösterreich, die wie die gleichen Befunde E. Müllers (Marburg) die Erfahrungen Wickmans vollauf bestätigten.

Ich bin absichtlich auf die Poliomyelitis-Epidemiologie und ihre Geschichte näher eingegangen, weil ihre Kenntnis m. E. für die Beurteilung der bisher vorliegenden widerspruchsvollen klinisch-epidemiologischen Erfahrungen bei der E. l. unerläßlich ist und uns als ein sehr geeigneter Führer auf die Bedingungen und Verhältnisse hinweist, unter denen es uns auch bei der E. l. gelingen dürfte, die Frage der Kontagiosität auf einer möglichst breiten Grundlage von Beobachtungen behufs einer allgemein gültigen Entscheidung zu lösen. Die zur Zeit vorliegenden Veröffentlichungen über die E. l. sind überaus zahlreich, das ihnen zugrunde liegende Material erstreckt sich auf viele Tausende von Fällen, wobei an weitaus erster Stelle Abhandlungen über die Klinilk der E. 1. stehen. Die Mehrzahl der größeren Arbeiten stammt aus Kliniken und Krankenhäusern, ihr Material aus größeren Städten und industriereichen, dichtbevölkerten Bezirken - nach obigen Ausführungen ein wesentlicher Umstand, der uns die negativen Ergebnisse hinsichtlich der Kontagiosität der E. l. erklären kann, wobei auch nicht außer acht gelassen werden darf, daß von den meisten Autoren die Frage der Kontagiosität überhaupt nicht berührt wird. Die bisher vorliegenden positiven Ergebnisse verpflichten uns jedenfalls, dieser epidemiologischen Frage der E. l. unser besonderes Augenmerk zu schenken; gilt dies insbesondere zur Zeit einer herrschenden Epidemie, so müssen wir doch auch die prolongierten Formen im Auge behalten, die namentlich zur Zeit eines akuten Schubes als Infektionsquelle in Betracht kommen können. Kling und Liljenquist konnten anläßlich einer E. 1.-Epidemie in einem einsamen Kirchspiel Schwedens den Nachweis der Kontagiosität in mehreren Fällen erbringen und haben hierüber ,vorläufig" berichtet; vielleicht gelingt es ihnen wie seinerzeit ihrem Landsmanne Wickman bei weiterer Nachforschung ein großes heweiskräftiges Material zu sammeln!

Als Gründe gegen die Kontagiosität der E. 1. wurde von verschicdenen Autoren angeführt, daß keine Krankenhausepidemien vorkommen, in ein und derselben Familie nur eine Person erkrankte, Encephalitiskranke zu anderen Patienten gelegt werden könnten, ohne daß dieselben hierdurch gefährdet würden - die gleichen Gründe, die man seinerzeit auch gegen die Kontagiositätslehre der Poliomyelitis ins 
Treffen geführt hat, die aber, wie wir wissen, keinen strikten Gegenbeweis bilden. Ich selbst sah gelegentlich der Poliomyelitisepidemie in Oberösterreich eine Reihe von Einzelerkrankungen in kinderreichen Familien und es ist mir nicht so selten untergekommen, daß erkrankte Kinder mit gesunden in ein und demselben Bette schliefen, ohne daß eine Infektion erfolgte; an eine Krankenhausinfektion kann ich mich bei der Poliomyelitis aus eigenen Erfahrungen nicht erinnern.

Können wir in den eigenen und den in der Literatur bereits zahlreich vorliegenden Beobachtungen von der unmittelbaren und indirekten Utbertragung der E. l. eine sichere Grundlage für die Kontagiositätslehre derselben erblicken, so haben wir außerdem noch andere Hinweise, welche hierfür zu sprechen scheinen. H. W. Maier fiel es auf, daß über die Hälfte seiner Fälle Eisenbahnangestellte und deren Angehörige sowie andere waren, die mit dem Fremdenverkehr in enger Berührung standen. Auch in unserem Material machen die Kranken, die beruflich in einem regen Verkehr mit der Mitwelt standen, die weitaus überwiegende Mehrheit der Gesamtfälle aus. Linz ist eine ziemlich rege Geschäftsstadt, die ebenso wie die übrigen größeren Städte der Provinz einen lebhaften Verkehr mit dem Lande unterhält; dazu kommt noch, daß die derzeit noch schwierige Beschaffung gewisser Lebensmittel die städtischen Bewohner zwingt, die bäuerliche Bevölkerung in ihren Höfen aufzusuchen. Das Land selbst weist zufolge eines ausgedehnten Netzes von Straßen und Eisenbahnen, der schiffbaren Donau einen regen Innen- und Durchzugsverkehr auf, wodurch die Verschleppung der Erkrankung zweifellos gefördert wird. Ich erinnere mich eines Eisenbahnbeamten, der Mitte Februar 1920 zu einer Konferenz nach Wien fuhr, sich dort 12 Tage aufhielt und 2 Tage nach seiner Heimkehr die ersten Anzeichen einer typischen E. 1. bot - Beispiele von Einschleppung der Erkrankung, wie wir sie bei der Poliomyelitis wiederholt gesehen haben.

Über gehäuftes Auftreten der E. 1. in hestimmten Gegenden des Landes - von größeren Städten abgesehen - konnte ich trotz der allerdings etwas spät eingeführten Anzeigepflicht keine verwertbaren Angaben bisher erhalten; es scheint die Neigung zur Bildung von Krankheitsherden bei der E. l. entschieden geringer zu sein wie bei der Poliomyelitis, was wohl ebenso wie die selteneren Beobachtungen von familiären Fällen für eine geringere Kontagiosität der E. l. sprechen würde. Immerhin fiel uns bei der Eintragung der einzelnen E. l.-Fälle hinsichtlich ihres Wohnsitzes in den Häuserplan der Stadt Linz eine mehr umsichriebene Häufung von Fällen in bestimmten Stadtvierteln und benachbarten Straßenzügen auf.

Vor Ausbruch der Poliomyelitisepidemie in Oberösterreich (1908/09) konnte man an verschiedenen Orten ein häufigeres Vorkommen der 
bereits als endemisch geltenden sporadischen Formen, selbst die Bildung kleiner Herde feststellen. Hinsichtlich der E. 1. machten wir ähnliche Beobachtungen, die wiederum für die enge epidemiologische Verwandtschaft der beiden Erkrankungen sprechen dürften; wir sahen - wie auch andere Autoren (Strü mpell) - in den letzten Jahren mehrere Fälle schwerer cerebraler Erkrankung, die wir nach unseren heutigen Erfahrungen über die E. 1. mit aller Wahrscheinlichkeit als solche ansprechen müssen; sogar die Bildung eines kleinen Herdes (die früher erwähnte Beobachtung Spechtenhausers) wurde beobachtet.

Unsere Beobachtungen lehren uns, daß die Encephalitis. lethargica epidemica von Mensch zu Mensch übertragbar ist, wobei die Ansteckung durch direkten Kontakt (durch nachweisbar Infizierte) und durch dritte Personen (klinisch anscheinend gesunde Viruszwischenträger) erfolgen kann. Nach den bisherigen Beobachtungen scheint die Kontagiosität der E. l. an sich gering und geringer zu sein als die der epidemischen Kinderlähmung.

\section{Literaturverzeichnis.}

1) Bakke, Ưber Encephalitis lethargica. Med. Rev. 38, Nr. 2; ref. in Zeitschr. f. d. ges. Neurol. u. Psychiatr. 25, H. 7. 1921. - 2) Claude und de Laulerie, Deux cas d'encéphalite épidémique survenus dans le milieu hospitalier. Bull. et mém. de la soc. méd. des hôp. de Paris 36, Nr. 41. 1921; ref. in Zeitschr. f. d. ges. Neurol. u. Psychiatr. 25, H. 7. 1921. $-{ }^{3}$ ) Co mby, Les encéphalites aiguës chez enfants. Bull. de la soc. de péd. de Paris 19, Nr. l; ref. in Zeitschr. f. d. ges. Neurol. u. Psychiatr. 26, H. 3. 1921. - 4) Do pter, La contagiosité de l'encéphalite épidémique. Paris méd. 11, Nr. 23. 1921; ref. in Zeitschr. f. d. ges. Neurol. u. Psychiatr. 26, H. 3. 1921. - ${ }^{5}$ ) Dopter, Aussprache zu Kling-Liljenquist (9). $\left.{ }^{6}\right)$ v. Economo, Die Encephalitis lethargica. Jahrb. f. Psych. 38. 1917. 7) v. Economo, Die Encephalitis lethargica-Epidemie von 1920. Wien. klin. Wochenschr. 1920, Nr. 16 u. 17. - ${ }^{8}$ ) Grünewald, Encephalitis epidemica (Sammelreferat). Zeitschr. f. d. ges. Neurol. u. Psychiatr. 25, H. 4. 1921. ${ }^{9}$ ) Kling und Liljenquist, Versuch der Feststellung der Verbreitungsweise der sog. Encephalitis letharg. (Vorläufige Mitt.). Svenska Läkartidningen 18, Nr. 8. 1921; ref. in Zeitschr. f. d. ges. Neurol. u. Psychiatr. 25, H. 6. 1921. 10) Lemierre, Encéphalite épidémique familiale. Transmission probable du virus par une personne atteinte depuis près de trois ans. Bull. et mém. de la soc. méd. des hôp. de Paris 36, Nr. 41. 1921; ref. in Zeitschr. f. d. ges. Neurol. u. Psychiatr. 25, H. 4. 1921. - 11) Le moine, Des relations étiologiques entre l'encéphalite léthargique et le hoquet épidémique. Ibidem 3\%, Nr. 9. 1921; ref. in Zeitschr. f. d. ges. Neurol. u. Psychiatr. 25, H. 7. 1921. - 12) H. W. Maier, Über die Encephalitis lethargica und ihr Auftreten in Zürich im Januar-Februar 1920. Schweiz. med. Wochenschr. 1920, Nr. 12. - ${ }^{13}$ ) Müller, E., Über die epidemische Encephalitis. Dtsch. Zeitschr. f. Nervenheilk. ro, H. 4-6. 1921. — 14) Müller, E., Epidemische Encephalitis unter dem Bilde rheumatischer Facialislähmung. Ref. in Zeitschr. f. d. ges. Neurol. u. Psychiatr. 26, H. I. 1921. - ${ }^{15}$ ) Müller, E., Die spinale Kinderlähmung. Springer, Berlin 1910. _- 16) Netter, Sur l'étiologie et la prophylaxie de l'encéphalite léthargique, sa déclaration obligatoire. Bull. 
de l'acad. de méd. 85, Nr. 10. 1921; ref. in Zeitschr. f. d. ges. Neurol. u. Psychiatr. 25, H. 6. 1921. - 17) Netter, Origine commune du hoquet épidémique et de l'encéphalite léthargique; relations chronologiques. Succession des ces manifestations chez le même sujet ou chez deux personnes en rapport entre elles. Bull. et mém. de la soc. méd. des hôp. de Paris 3r, Nr. 2. 1921; ref. in Zeitschr. f. d. ges. Neurol. u. Psychiatr. 25, H. 6. 1921. $-{ }^{18}$ ) Pecori, L'encefalite epidemica a Roma (1919/20). Dati epidemiologici e considerazioni. Ann. d'ig. 31, Nr. 1. 1921; ref. in Zeitschr. f. d. ges. Neurol. u. Psychiatr. 25, H. 7. 1921. - ${ }^{19}$ ) Piotrowski, L'encéphalite épidémique à Genève en 1920. Schweiz. med. Wochenschr. 1921, Nr. 6. - $\left.{ }^{20}\right)$ Roger, Réveil hivernal de l'épidémie encéphalitique et reviviscence saisonnière des encéphalites à forme prolongée. Bull. et mém. de la soc. méd. des hôp. de Paris 36, Nr. 41. 1921; ref. in Zeitschr. f. d. ges. Neurol. u. Psychiatr. 25, H. 4. 1921. - ${ }^{21}$ ) Roger und Blanchard, A propos de la contagion de l'encéphalite épidémique, deux cas survenus chez deux élèves caporaux. Ibidem 37, Nr. 2. 1921; ref. in Zeitschr. f. d. ges. Neurol. u. Psychiatr. 25, H. 6. 1921. 22) Römer, Die epidemische Kinderlähmung. Springer, Berlin 1911. _ 23) Schlesinger, Die jetzt in Wien herrschende Nervengrippe (Encephalitis, Polyneuritis und andere Formen). Wien. klin. Wochenschr. 1920, Nr. 17. - ${ }^{24}$ ) Stiefler, Zur Klinik der Encephalitis lethargica. Ibidem 1920, Nr. 14 u. 33. - ${ }^{25}$ ) Stiefler, Über hypophysäre Fettsucht als Restzustand eines Falles von Enceph. letharg. Monatsschr. f. Psychiatr. u. Neurol. 50, H. 2. 1921. - ${ }^{26}$ ) Stiefler und v. K urz, Über die Encephalitis letharg. epidem. (Erscheint demnächst in der Wien. med. Wochenschr.) - ${ }^{27}$ ) Stiefler, Über das Vorkommen der spinalen Kinderlähmung in Oberösterreich (1909-1913). Wien. klin. Wochensehr. 1915, Nr. 40. 28) v. Strü m pell, Über Encephalitis epidemica (Enceph. letharg.). Dtsch. med. Wochenschr. 1920, Nr. 26. - ${ }^{29}$ ) U m ber, Über Mesencephalitis epidemica (Enceph. letharg.). Ibidem 1921, Nr. 10. — ${ }^{30}$ ) Wickman, Die akute Poliomyelitis (HeineMedinsche Krankheit). Handbuch der Neurologie (Lewandowsky), Bd.2, S. 807. 